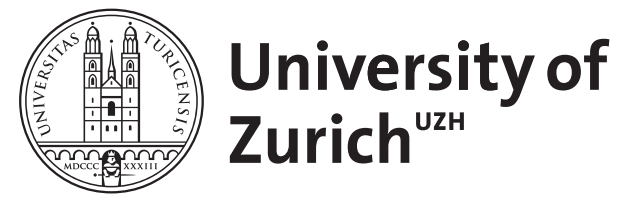

Die gesellschaftliche Verteilung von Traurigkeit. Eine emotionssoziologische Analyse

Jakoby, Nina

DOI: https://doi.org/10.1007/s11614-013-0068-4

Posted at the Zurich Open Repository and Archive, University of Zurich ZORA URL: https://doi.org/10.5167/uzh-85962

Journal Article

Published Version

Originally published at:

Jakoby, Nina (2013). Die gesellschaftliche Verteilung von Traurigkeit. Eine emotionssoziologische Analyse. Österreichische Zeitschrift für Soziologie, 38(1):33-53.

DOI: https://doi.org/10.1007/s11614-013-0068-4 


\title{
iözs-
}

\section{Die gesellschaftliche Verteilung von Traurigkeit. Eine emotionssoziologische Analyse}

\author{
Nina R. Jakoby
}

Zusammenfassung: Die Soziologie interessiert sich für die soziale Verteilung und Determinanten von Traurigkeit als Teil der Gefühlskultur einer Gesellschaft. Sie eröffnet zugleich Potenziale für das Themenfeld der sozialen Ungleichheit von Emotionen. Auf der Grundlage einer Querschnittsanalyse von Daten des Schweizer Haushalt-Panels (SHP) werden Zusammenhänge zwischen der Häufigkeit der erlebten Traurigkeit und Merkmalen der sozialstrukturellen Position, des sozialen Kontextes sowie in Abhängigkeit von kritischen Lebensereignissen geprüft. Der Beitrag bestätigt Variationen von Traurigkeit entlang sozialer Merkmale wie Geschlecht, Einkommen, Alter, Nationalität und Sprachregion.

Schlüsselwörter: Traurigkeit · Emotion · Sozialstruktur · Soziale Ungleichheit ·

Schweizer Haushalt-Panel

\section{The societal distribution of sadness. An analysis from a sociology of emotions perspective}

\begin{abstract}
Sociology is interested in the social incidence of and the factors that account for sadness as a part of a society's culture of emotions. At the same time, it opens a range of opportunities for the study of social inequality in experiencing emotions. Based on Swiss Household Panel (SHP) data, relationships are assessed between the frequency of sadness, and the parameters of one's position in the social structure, the social context, as well as critical life events. The paper confirms that sadness is reported differently along social predictors like gender, income, age, nationality and language region.
\end{abstract}

Keywords: Sadness $\cdot$ Emotion $\cdot$ Social structure $\cdot$ Inequality $\cdot$ Swiss Household Panel

(C) Springer Fachmedien Wiesbaden 2013

Dr. phil. N. R. Jakoby $(\bowtie)$

Soziologisches Institut, Universität Zürich, Andreasstrasse 15,

8050 Zürich, Schweiz

E-Mail: jakoby@soziologie.uzh.ch 


\section{Einleitung ${ }^{1}$}

Seit den 1990er Jahren ist ein zunehmendes Interesse an Emotionen als soziologischem Forschungsgegenstand feststellbar. Folge dieses emotional turn (vgl. Hopkins et al. 2009) ist eine verstärkte Reflexion über Emotionen als sozialen Tatbestand. Dennoch sind verschiedene Forschungsdefizite zu konstatieren. Hierzu zählen insbesondere die Vernachlässigung der theoretischen und empirischen Analyse von spezifischen Emotionen sowie die quantitative Analyse der sozialen Korrelate von Emotionen (vgl. Thoits 1989; Turner und Stets 2005). Die Emotion Traurigkeit steht im Vordergrund dieses Beitrages. Wie sich Traurigkeit in einer Gesellschaft verteilt und welchen sozialen Bedingungen sie unterliegt, sind weitgehend offene empirische Fragen. Sie stehen jedoch im Zentrum einer soziologischen Emotionsforschung, die Rückschlüsse auf soziale Ungleichheiten im emotionalen Erleben ermöglicht (vgl. Turner und Stets 2005) und das emotionale Klima (vgl. Vester 1991) einer Gesellschaft beschreiben kann. Die soziologische Perspektive auf Emotionen erfordert dabei eine Abgrenzung zu pathologischen Formen von Traurigkeit (z. B. depressiver Störung), wie sie Thoits (1989) bereits Ende der 1980er Jahre beschrieben hat:

[...] we lack basic information about the ,epidemiology ${ }^{6}$ of emotions, both in our own culture and across cultures. We hold many beliefs about the frequencies and distributions of emotional experiences, but these have not been tested systematically. [...] The frequencies and distributions of various feelings across social positions (particularly, everyday ' emotions rather than pathological states such as depression or phobias) are basic and necessary pieces of data for building a sociological theory of emotions. (Thoits 1989, S. $321 \mathrm{f}$.)

Die Soziologie interessiert sich für die gesellschaftliche Verbreitung und soziale Variationen von Traurigkeit. Folgende Fragen stehen im Vordergrund der Studie: Wie verteilt sich Traurigkeit entlang der sozialstrukturellen Position von Individuen? Welche sozialen Korrelate des individuellen Gefühls Traurigkeit gibt es? Zeigen sich soziale RegelmäBigkeiten in der Häufigkeit des Traurigkeitsempfindens in Bezug auf Geschlecht, Alter, sozioökonomischen Status, Familienstand und andere soziale Merkmale? Der Fokus der Analyse liegt damit auf den sozialen Bedingungen von Traurigkeit; sie eröffnet zugleich Potenziale für das Themenfeld der sozialen Ungleichheit von Emotionen.

Im Einzelnen werden aus soziologischen Emotionstheorien und verwandten theoretischen Ansätzen generierte Hypothesen über Zusammenhänge zwischen Traurigkeit und sozialstrukturellen Merkmalen sowie kritischen Lebensereignissen geprüft. Damit wird erstmals eine integrierte theoretische Perspektive auf die Emotion Traurigkeit entwickelt, die in Erweiterung der dominierenden psychologischen Forschung mehr als nur die klinische Analyse dieses Gefühls erfasst. Im zweiten Abschnitt erfolgt zunächst eine Begriffsbestimmung der Emotion Traurigkeit. Im dritten Abschnitt wird der theoretische Hintergrund der Studie beschrieben. Neben zwei prominenten emotionssoziologischen

1 Diese Studie wurde mit Daten des Schweizer Haushalt-Panels (SHP), das vom Schweizer Kompetenzzentrum Sozialwissenschaften (FORS) geleitet wird, durchgeführt. Das Projekt wird vom Schweizerischen Nationalfonds zur Förderung der wissenschaftlichen Forschung finanziert. 
Ansätzen (Kemper, Hochschild) werden die zentralen Aussagen der soziologischen Stresstheorie und der Theorie des subjektiven Wohlbefindens für die Erklärung von Traurigkeit skizziert. Im vierten Abschnitt folgt ein Überblick über den empirischen Forschungsstand. Im Anschluss hieran werden uni-, bi- und multivariate Analysen auf der Grundlage einer Querschnittsanalyse des Schweizer Haushalt-Panels dargestellt. Die Daten ermöglichen es, repräsentative Aussagen über die Häufigkeit und Verteilung von Traurigkeit zu treffen und zu diskutieren. Der Beitrag schließt mit einem zusammenfassenden Fazit.

\section{Zum Verständnis von Traurigkeit}

Traurigkeit wird übereinstimmend zu den Primär- bzw. Basisemotionen gezählt (vgl. Vester 1991; Turner und Stets 2005; Turner 2007; Bonanno et al. 2010). Dem emotionalen Komplex „Traurigkeit“ werden u. a. Trauer, Depressionen und Einsamkeitsgefühle zugeordnet (Vester 1991, S.33). Sie ist eine negative, schmerzhafte Emotion und wird von der Depression klar abgegrenzt, auch wenn Traurigkeit eine affektive Komponente der Depression darstellt (vgl. Mirowsky und Ross 1989; Leventhal und Martell 2006; Horwitz und Wakefield 2007). Traurigkeit kann sich dabei auf verschiedene Arten ausdrücken, von leichten Formen bis hin zu schweren Erscheinungen (Vester 1991, S. 158). Die Definition einer Depression als „,verstärktes Traurigkeitssyndrom“ (Vester 1991, S. 159) erfolgt rein symptombasiert. Das Diagnostic and Statistical Manual of Mental Disorders (DSM-IV) versteht unter einer Depression alle Zustände, die mindestens fünf von neun Symptomen aufweisen, u. a. Niedergeschlagenheit, Konzentrationsprobleme, Freudlosigkeit, Gewichtsverlust, Schlafstörungen oder Müdigkeit und mindestens 2 Wochen andauern (Horwitz und Wakefield 2007, S. 105-107). Traurigkeit bezieht sich zum einen auf Erfahrungen innerhalb der zuvor beschriebenen Symptomatik der Depression. Hierzu zählen beispielsweise die (ereignisunabhängige) gedrückte Grundstimmung und Niedergeschlagenheit. Zum anderen werden konkrete (Verlust-)Ereignisse als Auslöser für Traurigkeit angesehen. Die von Horwitz und Wakefield (2007) beschriebene Kategorie der normalen Traurigkeit (normal sadness) ist definiert als eine Reaktion auf Veränderungen des sozialen und individuellen Kontextes. Traurigkeit wird von drei situationsbedingten Quellen des Verlustes ausgelöst: „losses of attachment, status and meaning“" (Horwitz und Wakefield 2007, S. 43). Zum einen beziehen sich Verlustereignisse auf soziale und intime Beziehungen, wie Freundschaft oder Liebe (vgl. Bowlby 1980; Bonanno et al. 2010). Darüber hinaus kann sich der Verlust auf hierarchische Aspekte von Sozialbeziehungen beziehen, im Speziellen auf Dimensionen von Macht, Status, Respekt, Ressourcen oder Prestige. Hierunter fällt beispielsweise ein Status- oder Machtverlust durch Arbeitslosigkeit oder Berentung. Eine dritte Kategorie von Verlusten nimmt Bezug auf ein Scheitern im Erreichen von Zielen oder Idealen, die als wichtig im Leben erachtet werden. Weiterhin werden Traurigkeitsgefühle in einen unmittelbaren Zusammenhang mit chronischen Belastungen und Stress gebracht. Hierunter fallen ökonomische und soziale Belastungen, erlittene Ungerechtigkeiten oder dauerhafte Konflikte (Horwitz und Wakefield 2007, S. $27 \mathrm{f}$.). 
In dem folgenden Beitrag wird Traurigkeit als Emotion verstanden, die sich sowohl auf die ereignisunabhängige Niedergeschlagenheit und gedrückte Grundstimmung, welche nicht unmittelbar auf einen Verlust zurückzuführen ist, als auch auf situationsspezifische (bewusst oder unbewusst wahrgenommene) Auslöser beziehen kann. Ziel der Untersuchung ist es, die Häufigkeit und Verteilung des ,alltäglichen“ Gefühls der Traurigkeit auf der Grundlage von Selbstauskünften innerhalb einer Bevölkerungsumfrage zu analysieren. Aus diesem Grund können die kognitiven Bewertungen von konkreten Ereignissen, auf denen die situations- und verlustspezifischen Definitionen von Traurigkeit basieren, nicht Gegenstand der empirischen Analyse sein. Zudem muss zwischen dem Erleben und Ausdruck von Emotionen differenziert werden (vgl. auch Brody 1997). Survey-Daten messen nicht das tatsächliche Empfinden von Emotionen, sondern den verbalen Ausdruck bzw. das Kommunizieren von Traurigkeit. Diese zwei Analyseebenen lassen sich jedoch mit quantitativen Methoden nicht differenzieren.

\section{Theoretischer Hintergrund}

Da Traurigkeit ein theoretisches und empirisches Forschungsdesiderat der Soziologie allgemein und speziell im Feld der Emotionssoziologie ist, wird die Betrachtung auf verwandte Themengebiete erweitert. Neben emotionssoziologischen Ansätzen von Hochschild $(1979,2006)$ und Kemper $(1981 a, b)$ wird auf die soziologische Stresstheorie und das Konzept des subjektiven Wohlbefindens zurückgegriffen. Diese Vorgehensweise ermöglicht es, theorievergleichend die unterschiedlichen sozialen Korrelate der Traurigkeit herauszuarbeiten.

\subsection{Emotionssoziologische Ansätze: Kemper und Hochschild}

Die Sozialstruktur ist nach Kemper (1981a, S. 338) ein allgemeines Konzept, das sich sowohl auf die Makroebene (z. B. Organisationen, soziale Klassen) als auch auf die Mikroebene und die interpersonellen Beziehungen zwischen Akteuren bezieht. Das emotionale Erleben wird als eine Reaktion auf ein Interaktionsergebnis verstanden. Emotionen ergeben sich aus erlebten, vorgestellten oder erwarteten Ergebnissen sozialer Beziehungen (Kemper 1981b, S. 145). Wenn ein Individuum zu wenig Anerkennung aufgrund einer zu geringen Status-Macht-Position in Begegnungen mit anderen erhält, dann können sich negative Gefühle wie Enttäuschung, Ärger oder Traurigkeit einstellen (Flam 2002, S. 150). Macht bezieht sich auf, actions that are coercing, forcing, threatening, punishing, (...) producing thereby a relationship of domination and control of one actor by the other" (Kemper 1981a, S. 337). Status hingegen ist eine ,scalar dimension reflecting the amount of uncoerced, willing compliance, approval, deference, reward, praise, emotional or financial support, even love, that actors accord each other" (Kemper 1981a, S.337). Damit umfasst die Machtdimension sozialer Beziehungen Handlungen wie Gewalt, Zwang, Bedrohung oder Beherrschung, während die Statusdimension die freiwillige Gewährung von emotionaler Unterstützung, Liebe, Freundschaft oder sonstigen Belohnungen impliziert (Kemper 1981b, S. 139). 
Status loss by self (where self is agent in an irremediable sense, or where circumstances - Fate, God, Life, or other intractable force - is responsible) instigates sadness-depression. (Kemper 1991, S.334)

Die Theorie der negativen Gefühle nach Kemper postuliert damit einen Zusammenhang zwischen einer niedrigen Status-Macht-Position und dem häufigeren Erleben von negativen Emotionen. Dieses Erklärungsmodell lässt sich auch auf Ärger, Wut, Neid oder Scham anwenden (vgl. Turner und Stets 2005).

Thus, the structure of inequality will correspond to the distribution of positive and negative emotions, with higher-ranking individuals more likely to experience positive emotional arousal than lower-ranking persons, with the result that emotions become yet one more resource distributed unequally. (Turner und Stets 2005, S. 294)

Emotionen korrelieren mit der sozialstrukturellen Position von Individuen im sozialen Raum (vgl. Simon und Nath 2004; Turner und Stets 2005). Von Scheve (2009, 2012) diskutiert die verschiedenen Mechanismen, die den Einfluss des sozioökonomischen Status auf die erlebten Emotionen erklären können. Im Vordergrund der theoretischen Argumentation stehen dabei die strukturellen Grundlagen von Einschätzungen (von Scheve 2009, S. 160) bzw. die Abhängigkeit kognitiver von sozialen Strukturen (von Scheve 2012, S. 133). Prozesse der Emotionsentstehung sind offen für Einflüsse der sozialen Umwelt. So entstehen beispielsweise kognitive Strukturen, die einer Einschätzung zugrunde liegen, zu großen Teilen in Sozialität und enthalten bereits Regelmäßigkeiten der sozialen Umwelt, die sich in den Emotionen wiederfinden (von Scheve 2009, S. 144). Akteure wachsen in mehr oder weniger stabilen sozialen Strukturen auf, die sich durch eine gewisse Homogenität oder Präsenz von bestimmten Schlüsselereignissen und -erlebnissen auszeichnen. Aus diesem Grund erscheint es plausibel, dass ein Großteil der Akteure über vergleichbare (implizite und explizite) Erinnerungen und Wissensbestände, z.B. Erfahrungen mit Institutionen, die sich innerhalb bestimmter sozialstruktureller Zusammenhänge ähneln, verfügt, die sich dann in den Emotionen widerspiegeln (von Scheve 2009, S. 152). Niedrige Status- und sozioökonomische Positionen sind mit hoher Wahrscheinlichkeit mit finanziellen Restriktionen und höheren Arbeitsbelastungen (z. B. Arbeitsplatzunsicherheit) verbunden, die sich auf das emotionale Wohlbefinden auswirken (Horwitz und Wakefield 2007, S. 202). Darüber hinaus geht ein niedriger sozioökonomischer Status mit hoher Wahrscheinlichkeit damit einher, Situationen von Status- oder Kontrollverlust, chronischen Belastungen sowie ein Scheitern von Zielen zu erleben, die innerhalb der (ereignisorientierten) Definition von Traurigkeit beschrieben werden (vgl. Horwitz und Wakefield 2007; Vester 1991).

In diesem Kontext weist Hochschild $(1979,2006)$ auf eine weitere Verbindung zwischen der Sozialstruktur und Traurigkeit hin. Beide sind über soziale Normen und Gefühlsregeln vermittelt. Nach Badura und Pfaff (1989, S. 648) liefert Hochschild zum einen eine Mikrosoziologie der Gefühlsmanifestation, des Gefühlsmanagements und -ausdrucks von Individuen und zum anderen eine Makrosoziologie sozialer Gefühlsregeln. Gefühlsregeln spezifizieren, welche Gefühle wann und mit welcher Intensität und Dauer zum Ausdruck gebracht werden (Flam 2002, S. 130). Das Erleben und der 
Ausdruck von Traurigkeit sind durch Gefühlsnormen (feeling rules) und Expressionsnormen (display rules) geprägt (Thoits 1989, S. 322). Hochschild (2006, S. 73 f.) weist in diesem Zusammenhang auf eine gesellschaftlich differenzierte Verteilung von Gefühlsregeln für Männer und Frauen hin, die stereotypen Vorstellungen von männlichen und weiblichen Emotionen entsprechen. Im Allgemeinen werden Geschlechterunterschiede im emotionalen Erleben und Ausdruck als eine Funktion von Geschlechterrollenerwartungen, Status-Macht-Unterschieden oder der affektiven Sozialisation betrachtet (Brody 1997, S.386). In der Diskussion um Geschlechterdifferenzen im emotionalen Erleben und Ausdruck müssen ebenfalls die Modalitäten der gemessenen Emotionen, z. B. Intensität oder Häufigkeit, Selbstauskünfte oder physiologische Messungen, Beobachtung von Verhalten oder Mimik, berücksichtigt werden (Brody 1997, S. 370 f.). An dieser Stelle ist eine Unterscheidung zwischen dem Ausdruck und dem Erleben von Traurigkeit von Frauen und Männern von zentraler Bedeutung (vgl. Mirowsky und Ross 1995; Simon 2002; Simon und Nath 2004). Traurigkeit wird als weiblich konnotierte Emotion betrachtet und auf geschlechtsspezifische Gefühls- und Expressionsnormen verwiesen (vgl. Brody 1997; Simon und Nath 2004; Simon 2002). Studien zeigen, dass bei Kontrolle der emotionalen Expressivität der Geschlechterunterschied in der Häufigkeit der empfundenen Traurigkeit bestehen bleibt (vgl. Mirowsky und Ross 1995). Aus diesem Grund wird angenommen, dass Frauen häufiger Traurigkeit erleben und dieses Gefühl auch häufiger kommunizieren:

$[\ldots]$ as our culture contains norms that discourage men from feeling and expressing sadness $[\ldots]$, we should also find that women report that they experience and express sadness more often than men [...]. (Simon und Nath 2004, S. 1139)

Ein weiterer Erklärungsansatz fokussiert auf geschlechtsspezifische (emotionale) Reaktionen auf Stressoren, die sich bei Frauen in der Internalisierung emotionaler Probleme und damit Depressionen äußern, während es bei Männern überwiegend zu einer Externalisierung dieser Probleme in Form von Substanzmissbrauch, z. B. Alkohol, oder antisozialem Verhalten kommt (vgl. Aneshensel et al. 1991; Simon 2002). In Übereinstimmung mit geschlechtsspezifischen Stereotypen und Expressionsnormen werden Gefühle der Traurigkeit von Männern eher unterdrückt (vgl. Brody 1997).

\subsection{Soziologische Stresstheorie, Ressourcenkonzept und subjektives Wohlbefinden}

Im Vordergrund der soziologischen Stresstheorie (vgl. Pearlin 1989; Aneshensel et al. 1991) stehen drei Aspekte, die für die Erklärung von Traurigkeit von besonderer Bedeutung sind. Kritische Lebensereignisse werden als Stressoren und „,exogene Schocks“ (vgl. Ormel et al. 1999) bezeichnet, die in einem negativen Zusammenhang mit der psychischen Gesundheit bzw. dem subjektiven Wohlbefinden stehen (vgl. Filipp 1981). Hierzu gehören Verlustereignisse, wie zum Beispiel der Tod Nahestehender oder Ehescheidung. Ebenso zählen chronische Belastungen zu den Stressoren, d.h. primär Krankheiten oder dauerhafte Konflikte. Die Bewältigung ist abhängig von sozialen (externen) Ressourcen, die die Vulnerabilität der Individuen gegenüber Stressoren beeinflussen. Der Zugang zu sozialen Netzwerken und die damit verbundenen emotionalen und praktischen Unterstützungsleistungen sind zentrale interpersonelle Ressourcen (vgl. Stroebe und Stroebe 
1987; Stylianos und Vachon 2006). Im Einzelnen werden der Einfluss von unterschiedlichen Strukturmerkmalen von Netzwerken (Dichte, Größe, Häufigkeit) und die Qualität der Sozialbeziehungen und Unterstützungsleistungen analysiert (vgl. zusammenfassend Mirowsky und Ross 1986; Hurrelmann 2000; Stylianos und Vachon 2006). Riches und Dawson (2000, S. 18) nennen soziale Ressourcen wie stabile emotionale Beziehungen, Kommunikationsmöglichkeiten oder verfügbare Sozialbeziehungen außerhalb der Familie. Zusätzlich werden der Gesundheitsstatus und materielle Ressourcen (Einkommen) angeführt (vgl. Ormel et al. 1999). Auch die soziale Integration in Vereinen und anderen Organisationen steht in einem negativen Zusammenhang mit Traurigkeit oder Depressionen. Die Mitgliedschaft in formellen Vereinen oder anderen Gruppen ist ein Indikator für eine externe soziale Integration der Individuen. Regelmäßige und auf Dauer angelegte Beziehungen im Verein bieten gemeinschaftsbezogene Kommunikations- und Hilfepotenziale (vgl. Freitag 2004). Der Heiratsstatus gilt ebenfalls als ein Indikator für soziale Integration, der einen negativen Einfluss auf Traurigkeit und negative Emotionen im Allgemeinen hat (vgl. Mirowsky und Ross 1986). Des Weiteren wird auf die ungleiche Verteilung von Ressourcen, Restriktionen und Opportunitäten im Erleben und Umgang mit Stress hingewiesen, d. h. den strukturellen Kontext, in den die individuelle Stressreaktion eingebettet ist (vgl. Pearlin 1989; Horwitz und Wakefield 2007). Ressourcen sind sozial selektiv verteilt, d.h. ihr Besitz variiert positiv mit dem sozialen Status (vgl. Mirowsky und Ross 1986; Badura und Pfaff 1989). So korrelieren beispielsweise der Bildungsstatus und andere Schichtindikatoren positiv mit der Netzwerkgröße (vgl. zusammenfassend Wolf 2004). Die Netzwerkgröße ihrerseits korreliert negativ mit depressiven Symptomen und positiv mit der Lebenszufriedenheit (vgl. Stylianos und Vachon 2006).

Eine soziologische Betrachtung der Emotion Traurigkeit muss ebenfalls das Konzept des subjektiven Wohlbefindens berücksichtigen. Im Kern des theoretischen Ansatzes von Ormel et al. (1999) auf Grundlage der Social Production Theory steht die Annahme, dass subjektives Wohlbefinden durch drei instrumentelle Ziele produziert werden kann. Hierzu zählen soziale Beziehungen und die damit verbundene Zuneigung, Liebe oder Freundschaft (affection), Verhaltensbestätigung (behavioural confirmation) sowie Ressourcen und deren Kontrolle (status) (Ormel et al. 1999, S. 68). In diesem Kontext tragen insbesondere das soziale Netzwerk und die Erwerbstätigkeit zum Erreichen dieser Ziele bei und stehen in einem positiven Zusammenhang mit dem subjektiven Wohlbefinden. Als Restriktionen für die Produktion von Wohlbefinden gelten fehlende (materielle und immaterielle) Ressourcen sowie kritische Lebensereignisse.

Die vorgestellten theoretischen Ansätze verdeutlichen den Zusammenhang zwischen Traurigkeit und der sozialstrukturellen Position der Individuen, der die Annahme der sozial ungleichen Verteilung der Emotion Traurigkeit innerhalb einer Gesellschaft verstärkt. Zudem muss auf die Verbindung zwischen der Stresstheorie und emotionssoziologischen Ansätzen hingewiesen werden (vgl. Lennon 1989). Diese besteht insbesondere zu Kempers (1981a, b) Status- und Machtpositionen, die vor allem durch Geschlecht, soziale Klasse und ethnische Herkunft konstituiert werden, und den Gefühlsregeln Hochschilds (1979, 2006), die Geschlechterunterschiede im Erleben und Ausdruck von Emotionen zu erklären vermögen. Im nächsten Abschnitt werden nun die empirischen Korrelate von Traurigkeit zusammengefasst und der gegenwärtige Forschungsstand skizziert. 


\section{Forschungsstand}

Traurigkeit ist - mit Ausnahme der Studie von Simon und Nath (2004) - nur sehr selten ein empirischer Forschungsgegenstand. Aus diesem Grund wird der Blick auf verwandte Forschungsfelder wie Indikatoren des subjektiven Wohlbefindens, z. B. Lebenszufriedenheit (vgl. Stevenson und Wolfers 2009) und psychische Beschwerden (vgl. Rüesch und Manzoni 2003), erweitert. Darüber hinaus ist das Gefühl der Traurigkeit Bestandteil des allgemeinen Konzeptes „distress“ (vgl. Mirowsky und Ross 1986, 1989, 1995). Distress als „unpleasant subjective state“ (Mirowsky und Ross 1989, S.21) stellt das Gegenteil von Wohlbefinden dar. Die Angaben über soziale Korrelate von Traurigkeit oder Indikatoren des subjektiven Wohlbefindens basieren auf Selbstauskünften von Individuen auf der Grundlage von Survey-Daten.

Den Forschungsstand über die Determinanten von distress zusammenfassend benennen Mirowsky und Ross (1989) vier robuste Faktoren: Geschlecht, sozialer Status, Heiratsstatus und kritische Lebensereignisse. Diese Determinanten können ebenfalls auf die Traurigkeit übertragen werden, wie der im Folgenden skizzierte Forschungsstand zeigt.

Eine der wenigen repräsentativen Studien, die auf der Grundlage des Emotionsmoduls im General Social Survey im Jahr 1986 explizit die Determinanten verschiedener Emotionen analysiert, ist die Studie von Simon und Nath (2004). Traurigkeit wird über die Anzahl der Tage in den letzten Wochen, in denen sich die Befragten traurig gefühlt haben, operationalisiert (Simon und Nath 2004, S. 1145). Unter Kontrolle von Statusmerkmalen und anderen Drittvariablen erweist sich der Geschlechtereffekt als stabil. Frauen geben an, häufiger trauriger zu sein als Männer (vgl. auch Mirowsky und Ross 1995). Darüber hinaus zeigen sich ein negativer Alters- und Bildungseffekt und ein signifikanter Einkommenseffekt. Jüngere und Personen mit niedrigem sozioökonomischen Status berichten über ein häufigeres Traurigkeitsempfinden. Weiterhin korrelieren die Erwerbstätigkeit und der Ehestatus negativ mit Traurigkeit. Simon und Nath (2004) bewerten ihre Befunde als Bestätigung der strukturellen Theorie Kempers. Mirowsky und Ross (1995) untersuchen zusätzlich den Einfluss von geschlechtsspezifischen Unterschieden im Ausdruck von Traurigkeit. Selbst unter Kontrolle des „expression index“ sowie von Indikatoren der emotionalen Distanziertheit (,I keep emotions to myself“) geben Frauen an, häufiger trauriger zu sein. Die Autoren folgern hieraus, dass Geschlechterunterschiede nicht als Artefakte angesehen werden sollten, die auf eine höhere Bereitschaft der Frauen, ihre Gefühle auszudrücken, oder auf sozial wünschenswertes Antwortverhalten zurückgeführt werden können (vgl. Mirowsky und Ross 1995).

Simon (2002) findet Zusammenhänge zwischen Depressionen - hier operationalisiert anhand von zwölf Items, die u.a. auch die Anzahl von Tagen erfassen, an denen die Befragten traurig waren - und dem Geschlecht. Diese bleiben unter Kontrolle von soziodemografischen Drittvariablen wie Heirats-, Erwerbs- und sozioökonomischem Status bestehen. Weiterhin zeigen sich negative Effekte von sozioökonomischem Status, Einkommen, Heirats- und Erwerbsstatus. Eine internationale Längsschnittstudie zu den verschiedenen Indikatoren des subjektiven Wohlbefindens (Glück, Lebenszufriedenheit) bestätigt den Geschlechtereffekt für negative Emotionen in den USA und verschiedenen europäischen Ländern (vgl. Stevenson und Wolfers 2009). Die verschiedenen Analysen zeigen übereinstimmend, dass Frauen angeben, weniger glücklich bzw. zufrieden zu sein, 
auch unter Kontrolle von relevanten Drittvariablen wie Erwerbs- und Bildungsstatus, Alter oder ethnischer Herkunft.

Als weiterer Einflussfaktor im Zusammenhang mit Glück und Wohlbefinden wird die Elternschaft diskutiert. Entgegen der alltagstheoretischen Annahme kann die bisherige Forschung überwiegend negative Effekte von Kindern auf das psychische Wohlbefinden nachweisen (vgl. McLanahan und Adams 1987, 1989; zusammenfassend Mirowsky und Ross 1989). Dieser Befund wird auf ökonomische Belastungen, welche mit einer Elternschaft verbunden werden, die Qualität der Ehe und Rollenkonflikte der Frau zurückgeführt. Emotionssoziologische Studien zeigen weiterhin, dass niedrige Statuspositionen mit dem verstärkten Erleben von negativen Emotionen wie Angst, Hoffnungslosigkeit, Wut und Traurigkeit einhergehen (vgl. Neckel 1999; Simon und Lively 2010). Zudem gibt es negative Zusammenhänge zwischen der Häufigkeit von psychischen Problemen und dem sozioökonomischen Status (vgl. Stroebe und Stroebe 1987; Yu und Williams 2006).

Darüber hinaus werden auf der Grundlage der Schweizerischen Gesundheitsbefragung 2002 negative Zusammenhänge zwischen leichten psychischen Beschwerden (z. B. Unausgeglichenheit, Pessimismus, Niedergeschlagenheit) und dem Alter festgestellt (vgl. Rüesch und Manzoni 2003). Zudem zeigt sich ein Geschlechtereffekt, denn Frauen sind davon häufiger betroffen. Weiterhin konnte ein Zusammenhang zwischen dem Erleben von kritischen Lebensereignissen und psychischen Problemen nachgewiesen werden (vgl. Rüesch und Manzoni 2003). Einen anderen Ansatz verfolgen Budowski et al. (2009), die den Einfluss von Partnerschaftsereignissen auf die psychische Gesundheit in der Schweiz untersuchen. Scheidung und Verwitwung stehen in einem negativen Zusammenhang mit der psychischen Gesundheit. Die Lebenslaufperspektive der psychischen Gesundheit hebt zudem den Einfluss sogenannter kumulierter Nachteile hervor, die in der Biografie der Individuen zu verorten sind, z. B. kritische Lebensereignisse wie die Scheidung der Eltern, finanzielle Probleme in der Jugendzeit oder Bildungsstatus der Eltern.

\section{Daten, Methode und Operationalisierung}

Im Folgenden werden Zusammenhänge zwischen der Häufigkeit von Traurigkeit und sozialstrukturellen Einflüssen sowie kritischen Lebensereignissen geprüft. Damit erfolgt eine systematische und integrierende Überprüfung der in der einschlägigen Literatur diskutierten sozialen Korrelate von Traurigkeit. So fokussieren Simon und Nath (2004) ausschließlich auf Statusmerkmale, Geschlecht, ethnische Herkunft oder Elternstatus. Wichtige andere Faktoren, die für die Erklärung von Traurigkeit als theoretisch relevant erachtet werden, insbesondere das Sozialkapital und Stressoren der externen Umwelt (kritische Lebensereignisse, chronische Belastungen), wurden bisher nicht überprüft. Diese Faktoren stellen zugleich wichtige Kontrollvariablen für die vermuteten sozialstrukturellen Zusammenhänge dar.

Ziel des vorliegenden Beitrages ist die Integration der in den verschiedenen theoretischen Ansätzen diskutierten Determinanten der Traurigkeit. Aus den im Abschnitt 3 vorgestellten theoretischen Überlegungen ergeben sich folgende allgemeine Hypothesen: 
H1: Je niedriger der sozioökonomische Status, desto häufiger wird über Traurigkeit berichtet.

H2: Frauen geben an, häufiger Traurigkeit zu empfinden als Männer.

H3: Je weniger Sozialkapital, desto häufiger wird über Traurigkeit berichtet.

H4: Kritische Lebensereignisse erhöhen die Häufigkeit von Traurigkeit.

Explorativ sollen Zusammenhänge zwischen Alter, Familienstand, Elternstatus, Nationalität, Sprachregion und der Häufigkeit von Traurigkeit untersucht werden. Zur Erklärung eines möglichen Alterseffektes können das aus der Stresstheorie stammende Ressourcenkonzept, die Disengagement Theory sowie die Life Course Theory herangezogen werden (vgl. auch Schieman 2006). Verschiedene Prognosen über das emotionale Befinden im Alter sind denkbar. Während die Life Course Theory auf das mit dem Alter verbundene häufigere Erleben von kritischen Lebensereignissen (Todesfälle) und chronischen Belastungen (Krankheiten) verweist, steht im Vordergrund der Disengagement Theory die Loslösung von Rollenbeziehungen (Berufsrollen), die als Stressoren negative Effekte auf das emotionale Wohlbefinden haben. Gleichzeitig hebt die Theorie der sozioemotionalen Selektivität (vgl. Carstensen 1991, 1992) die Variabilität von psychosozialen Ressourcen hervor, wobei ein effektiverer Umgang mit Emotionen, Toleranz und Selbstkontrolle im Alter erwartet wird (vgl. zusammenfassend Schieman 2006). Des Weiteren können Überlegungen zu einem generationsspezifischen Ausdruck von Traurigkeit angeführt werden. In Anlehnung an Winkel (2004) kann vermutet werden, dass jüngere Kohorten über andere verbale Ressourcen verfügen, Traurigkeit auszudrücken und offen zu kommunizieren. Eine Partnerschaft hat einen positiven Effekt auf das Erleben von Traurigkeit, wobei keine Unterschiede zwischen Personen, die verheiratet sind, und solchen, die in einer Partnerschaft leben, erwartet werden. Zum Einfluss der Elternschaft zeigt die bisherige Forschung uneindeutige empirische Befunde (siehe Abschnitt 4). Darüber hinaus kann eine höhere emotionale Belastung von Migrantinnen und Migranten vermutet werden. Der Prozess der Aus- und Wiedereingliederung ist mit erheblichen psychosozialen Belastungen verbunden, zum Beispiel aufgrund der Loslösung von sozialen Netzwerken und traditionellen Glaubenssystemen (Badura und Pfaff 1989, S.651). Die Häufigkeit und Verteilung von Emotionen in den Schweizer Sprachregionen wurde bisher noch nicht differenziert analysiert.

Das Hauptziel des Schweizer Haushalt-Panels (SHP) ist die Beobachtung des sozialen Wandels und insbesondere der Lebensbedingungen der Bevölkerung in der Schweiz. Das SHP ist eine jährlich wiederholte Panelstudie, die eine Zufallsstichprobe von Haushalten in der Schweiz begleitet und deren Mitglieder interviewt. Die Datenerhebung startete im Jahr 1999 mit einer Stichprobe von 5074 Haushalten beziehungsweise 12.931 Haushaltsmitgliedern. Aktuell umfasst die Datenbank des SHP Informationen zu den Jahren 1999 bis 2011.2 Das SHP des Jahres 2008 (Welle 10) enthält eine Emotionsskala, die Freude, Sorgen, Traurigkeit sowie Ärger erfasst. Diese Daten ermöglichen es, die emotionalen Konsequenzen unterschiedlicher sozialstruktureller Positionen zu untersuchen.

2 Vgl. www.swisspanel.ch/?lang=de. Zugegriffen: 22. Juni 2012. 
Tab. 1: Häufigkeit der Emotion Traurigkeit
Datenbasis: SHP 2008, eigene Berechnung

\begin{tabular}{lrc}
\hline & $\mathrm{n}$ & Prozent \\
\hline Nie $(0)$ & 372 & 5,4 \\
& 598 & 8,7 \\
& 1.560 & 22,6 \\
& 1.412 & 20,5 \\
& 996 & 14,5 \\
& 980 & 14,2 \\
& 384 & 5,6 \\
& 303 & 4,4 \\
& 224 & 3,3 \\
Immer $(10)$ & 30 & 0,4 \\
$\mathrm{n}$ & 31 & 0,5 \\
$\bar{x}=3,4, \mathrm{~s}=1,9$ & 6.890 & \\
\hline
\end{tabular}

Ziel der Querschnittsanalyse ist die Aufdeckung der sozialen Variationen in der emotionalen Erfahrung bzw. im Ausdruck von Traurigkeit in Abhängigkeit von individuellen und familialen Merkmalen sowie Indikatoren des sozialen Status. Um den Begrenzungen früherer Studien (z.B. Simon und Nath 2004) Rechnung zu tragen, wird zusätzlich der Einfluss des Sozialkapitals und kritischer Lebensereignisse auf die Häufigkeit von Traurigkeit analysiert. Damit erfolgt erstmals eine systematische Überprüfung der sozialen Korrelate von Traurigkeit.

Die Häufigkeit von Traurigkeit wird über eine 11-er Skala erfasst: Wie häufig етрfinden Sie normalerweise folgende Gefühle - Traurigkeit? (0 bedeutet „,nie “ und 10 „, immer“). Tabelle 1 zeigt die Verteilung der abhängigen Variable.

Neben dem Geschlecht und sechs Alterskategorien (18-29, 30-39, 40-49, 50-59, 60-69, 70 und älter) werden Kinder im Haushalt (unter 18 Jahren) als dichotome Variable erfasst $(0=$ nein, $1=\mathrm{ja})$. Der Partnerschaftsstatus wurde gruppiert in $1=$ verheiratet, $2=$ mit Partner/in und 3=Single. Somit können der Effekt des Heiratsstatus als auch der Effekt der Partnerschaft überprüft werden. Der sozioökonomische Status wird über zwei Indikatoren abgebildet: das logarithmierte Äquivalenzeinkommen (Nettohaushaltseinkommen/Jahr nach OECD-Standard) und den Bildungsstatus, wobei 1=niedrig (keine Ausbildung, obligatorische Ausbildung, allgemeinbildende Schule), $2=$ mittel (Berufslehre, Vollzeitberufsschule, Matura) und 3 =hoch (höhere Berufsausbildung, Fachhochschule, höhere Fachhochschule, Universität). In Anlehnung an die Operationalisierung von Simon und Nath (2004) wird die Erwerbstätigkeit als dichotome Variable erfasst $(0=$ nein, 1=ja). Die Nationalität bzw. ethnische Herkunft eines Individuums wird über drei verschiedene Typen abgebildet. Es wird zwischen der Nationalität „Schweiz“, der Mischform „Schweiz und eine zweite Nationalität“ sowie „,andere Nationalität“ differenziert (1=Schweiz, 2 =Schweiz und andere, $3=$ =Andere). Ebenfalls werden die Sprachregionen der Schweiz in die Analyse aufgenommen (1=deutsch, 2 =französisch, $3=$ italienisch). 
Als Kennzahl der sozialen Einbindung im engeren persönlichen Umfeld wurde ein Summenindex generiert, der die Größe des Gesamtnetzwerkes erfasst. Er setzt sich aus der jeweiligen Anzahl der engen Freundinnen und Freunde, Bekannten und Verwandten zusammen. Als Maß der externen sozialen Integration dient gemäß Freitag (2004) die Mitgliedschaft in Vereinen $(0=$ nein, $1=\mathrm{ja})$. Zur Überprüfung des Einflusses von kritischen Lebensereignissen werden drei Ereignisse in die Modelle aufgenommen, die als dichotome Variablen konzipiert sind $(0=$ nein, $1=$ ja):

- der Tod einer/eines signifikanten Anderen (,,Ist (seit dem letzten Interview) eine Person gestorben, wo Ihnen nahe gestanden ist? “),

- das Ende einer engen Beziehung (,,Ist es (seit dem letzten Interview) zum Abbruch einer engen und wichtigen Beziehung gekommen - z. B. Auflösung von einer Freundschaft, Trennung, Scheidung?"),

- chronische Krankheiten (,,Haben Sie eine chronische Krankheit oder ein gesundheitliches Problem, das schon länger dauert?").

\section{Soziale Variationen von Traurigkeit}

Im Folgenden wird zuerst die deskriptive Verteilung der Häufigkeit von Traurigkeit entlang von Mittelwertvergleichen vorgestellt. Danach folgt die Darstellung der multivariaten Modelle. Tabelle 2 zeigt die Verteilung der Emotion Traurigkeit sowie die Operationalisierungen der unabhängigen Variablen.

Bivariat zeigen sich signifikante Unterschiede in der Häufigkeit von Traurigkeit in Bezug auf Geschlecht, Alter, Partnerschaftsstatus, Bildungsniveau, Erwerbstätigkeit, Nationalität und Sprachregion. In einem zweiten Schritt erfolgt nun die multivariate Überprüfung der postulierten Zusammenhänge zwischen der sozialstrukturellen Position, Sozialkapital sowie kritischen Lebensereignissen mittels linearer Regression. Tabelle 3 zeigt das Gesamtmodell sowie die Teilmodelle für Frauen und Männer.

Die Modelle ergeben eine gute Varianzaufklärung von 10,4 Prozent für das Gesamtmodell, 9,1 Prozent für das Teilmodell Frauen und 8,2 Prozent für das Teilmodell Männer. Die Ergebnisse bestätigen die erwarteten Zusammenhänge zwischen dem sozialen Status und der Häufigkeit von Traurigkeit. In Übereinstimmung mit der strukturellen Theorie Kempers zeigen sich signifikante Einflüsse des sozioökonomischen Status (Einkommen und Erwerbstätigkeit) auf die Häufigkeit der berichteten Traurigkeit. Dieser Befund ist als Hinweis auf emotionale Konsequenzen von niedrigen Statuspositionen zu deuten, wie er für die USA nachgewiesen wurde (vgl. Simon und Nath 2004) und nun auch für die Schweiz bestätigt werden kann. An dieser Stelle besteht ebenfalls Anschlussfähigkeit an emotionssoziologische Studien, die verdeutlichen, dass niedrige Statuspositionen mit dem verstärkten Erleben von negativen Emotionen wie Angst, Hoffnungslosigkeit oder Wut einhergehen (vgl. Neckel 1999; Simon und Lively 2010). In allen Modellen zeigt sich zudem, dass das Geschlecht einen signifikanten Einfluss hat: Frauen geben an, häufiger Traurigkeit zu empfinden. Damit kann dieser Befund in Übereinstimmung mit amerikanischen Studien (z. B. Mirowsky und Ross 1986; Simon und Nath 2004) für die Schweiz bestätigt werden. Unter Kontrolle des sozioökonomischen Status, Merkmalen 
Tab. 2: Mittelwertvergleiche der Häufigkeit von Traurigkeit ${ }^{\mathrm{a}}$

\begin{tabular}{|c|c|c|c|}
\hline & $\bar{x}$ & $\mathrm{~s}$ & $\mathrm{n}$ \\
\hline \multicolumn{4}{|l|}{ Geschlecht*** } \\
\hline Männer & 3,1 & 2,0 & 3.026 \\
\hline Frauen & 3,7 & 1,9 & 3.864 \\
\hline \multicolumn{4}{|l|}{ Alter* } \\
\hline 18-29 Jahre & 3,3 & 1,7 & 992 \\
\hline 30-39 Jahre & 3,3 & 1,8 & 881 \\
\hline 40-49 Jahre & 3,4 & 2,0 & 1.510 \\
\hline 50-59 Jahre & 3,5 & 2,0 & 1.239 \\
\hline 60-69 Jahre & 3,5 & 2,1 & 951 \\
\hline 70 Jahre und älter & 3,5 & 2,3 & 801 \\
\hline \multicolumn{4}{|l|}{ Partnerschaftsstatus $* * *$} \\
\hline Verheiratet & 3,4 & 1,9 & 3.690 \\
\hline Mit Partner/in & 3,3 & 1,9 & 1.419 \\
\hline Single & 3,7 & 2,0 & 1.775 \\
\hline \multicolumn{4}{|l|}{ Kinder im Haushalt } \\
\hline $\mathrm{Ja}$ & 3,4 & 2,0 & 4.331 \\
\hline Nein & 3,5 & 1,9 & 2.559 \\
\hline \multicolumn{4}{|l|}{ Bildungsstatus*** } \\
\hline Niedrig & 3,6 & 2,1 & 1.608 \\
\hline Mittel & 3,4 & 2,0 & 3.205 \\
\hline Hoch & 3,2 & 1,9 & 2.077 \\
\hline \multicolumn{4}{|l|}{ Erwerbstätigkeit } \\
\hline Ja & 3,3 & 1,9 & 4.740 \\
\hline Nein & 3,6 & 2,2 & 2.149 \\
\hline \multicolumn{4}{|l|}{ Nationalität*** } \\
\hline Schweiz & 3,3 & 1,9 & 5.595 \\
\hline Schweiz und andere & 3,9 & 2,2 & 777 \\
\hline Andere & 3,7 & 2,2 & 480 \\
\hline \multicolumn{4}{|l|}{ Sprachregion*** } \\
\hline Deutschsprachige Schweiz & 3,2 & 1,9 & 4.897 \\
\hline Französischsprachige Schweiz & 4,0 & 2,1 & 1.772 \\
\hline Italienischsprachige Schweiz & 4,2 & 2,1 & 221 \\
\hline
\end{tabular}

Datenbasis: SHP 2008, eigene Berechnung

${ }^{\mathrm{a}}$ Die ausgewiesenen Mittelwerte $(\bar{x})$ und Standardabweichungen (s) sind aus Gründen der Übersichtlichkeit auf eine Stelle hinter dem Komma gerundet.

Mittelwertunterschiede sig. zum $* 5 \%, * * 1 \%, * * * 0,1 \%$-Niveau (t-Test, ANOVA)

des sozialen Kontextes und dem Einfluss von kritischen Lebensereignissen bleibt der Geschlechtereffekt stabil. Die Geschlechterdifferenzen können somit nicht durch Unterschiede im sozioökonomischen Status erklärt werden. Vielmehr deuten die Ergebnisse darauf hin, dass Traurigkeit ein sozial akzeptiertes Gefühl von Frauen ist und Frauen häufiger über dieses Gefühl sprechen (vgl. Brody 1997). 
Tab. 3: Determinanten der Traurigkeit ${ }^{\mathrm{a}}$

\begin{tabular}{|c|c|c|c|}
\hline & Gesamt & Frauen & Männer \\
\hline Geschlecht (Männer) & $\begin{array}{l}0,534 * * * \\
(0,069)\end{array}$ & - & - \\
\hline \multicolumn{4}{|l|}{ Alter (18-29) } \\
\hline 30-39 & $\begin{array}{l}0,083 \\
(0,123)\end{array}$ & $\begin{array}{r}-0,070 \\
(0,161)\end{array}$ & $\begin{array}{c}0,219 \\
(0,189)\end{array}$ \\
\hline $40-49$ & $\begin{array}{l}0,221 * \\
(0,118)\end{array}$ & $\begin{array}{c}0,208 \\
(0,162)\end{array}$ & $\begin{array}{c}0,193 \\
(0,175)\end{array}$ \\
\hline $50-59$ & $\begin{array}{l}0,307 * \\
(0,122)\end{array}$ & $\begin{array}{c}0,266 \\
(0,163)\end{array}$ & $\begin{array}{r}0,348^{*} \\
(0,187)\end{array}$ \\
\hline $60-69$ & $\begin{array}{c}0,007 \\
(0,131)\end{array}$ & $\begin{array}{l}0,003 \\
(0,175)\end{array}$ & $\begin{array}{r}-0,008 \\
(0,210)\end{array}$ \\
\hline 70 und älter & $\begin{array}{c}-0,391 * * \\
(0,150)\end{array}$ & $\begin{array}{r}-0,144 \\
(0,197)\end{array}$ & $\begin{array}{c}-0,644^{* *} \\
(0,249)\end{array}$ \\
\hline \multicolumn{4}{|c|}{ Partnerschaftsstatus (Verheiratet) } \\
\hline Mit Partner/in & $\begin{array}{c}0,048 \\
(0,095)\end{array}$ & $\begin{array}{c}0,013 \\
(0,127)\end{array}$ & $\begin{array}{c}0,056 \\
(0,143)\end{array}$ \\
\hline Single & $\begin{array}{l}0,251^{* * *} \\
(0,095)\end{array}$ & $\begin{array}{c}0,098 \\
(0,117)\end{array}$ & $\begin{array}{c}0,329 * \\
(0,167)\end{array}$ \\
\hline Kinder $<18$ Haushalt $(\mathrm{Ja})$ & $\begin{array}{r}-0,058 \\
(0,093)\end{array}$ & $\begin{array}{c}-0,202 \\
(0,127)\end{array}$ & $\begin{array}{c}0,113 \\
(0,136)\end{array}$ \\
\hline \multicolumn{4}{|l|}{ Bildungsstatus (Niedrig) } \\
\hline Mittel & $\begin{array}{r}-0,158 \\
(0,106)\end{array}$ & $\begin{array}{l}-0,225^{*} \\
(0,127)\end{array}$ & $\begin{array}{r}-0,010 \\
(0,188)\end{array}$ \\
\hline Hoch & $\begin{array}{r}-0,160 \\
(0,117)\end{array}$ & $\begin{array}{r}-0,170 \\
(0,148)\end{array}$ & $\begin{array}{r}-0,062 \\
(0,195)\end{array}$ \\
\hline $\begin{array}{l}\text { Netto-HH-Jahreseinkommen } \\
\text { (Äquiv., log.) }\end{array}$ & $\begin{array}{l}-0,235^{* *} \\
(0,077)\end{array}$ & $\begin{array}{l}-0,291 * * \\
(0,101)\end{array}$ & $\begin{array}{r}-0,156 \\
(0,116)\end{array}$ \\
\hline Erwerbstätigkeit (Ja) & $\begin{array}{l}0,280 * * \\
(0,105)\end{array}$ & $\begin{array}{c}0,222 * \\
(0,123)\end{array}$ & $\begin{array}{c}0,370 * \\
(0,184)\end{array}$ \\
\hline \multicolumn{4}{|l|}{ Nationalität (Schweiz) } \\
\hline Schweiz und andere & $\begin{array}{l}0,506^{* * *} \\
(0,101)\end{array}$ & $\begin{array}{l}0,520 * * * \\
(0,133)\end{array}$ & $\begin{array}{l}0,498 * * * \\
(0,156)\end{array}$ \\
\hline Andere & $\begin{array}{l}0,343^{* *} \\
(0,118)\end{array}$ & $\begin{array}{l}0,546^{* * *} \\
(0,153)\end{array}$ & $\begin{array}{c}0,162 \\
(0,179)\end{array}$ \\
\hline Größe Gesamtnetzwerk & $\begin{array}{c}-0,006^{* *} \\
(0,002)\end{array}$ & $\begin{array}{r}-0,005 \\
(0,003)\end{array}$ & $\begin{array}{c}-0,006^{*} \\
(0,003)\end{array}$ \\
\hline
\end{tabular}


Tab. 3: (Fortsetzung)

\begin{tabular}{lccc}
\hline & Gesamt & Frauen & Männer \\
\hline Mitglied Verein (Nein) & 0,035 & 0,070 & $-0,016$ \\
& $(0,069)$ & $(0,092)$ & $(0,101)$ \\
Tod Nahestehende/r (Nein) & $0,521^{* * *}$ & $0,510^{* * *}$ & $0,514 * * *$ \\
& $(0,083)$ & $(0,112)$ & $(0,124)$ \\
Ende einer Beziehung (Nein) & $0,406^{* * *}$ & $0,414^{* * *}$ & $0,422^{*}$ \\
& $(0,121)$ & $(0,137)$ & $(0,212)$ \\
Chronische Krankheit (Nein) & $0,345^{* * *}$ & $0,374 * * *$ & $0,292^{* *}$ \\
& $(0,071)$ & $(0,374)$ & $(0,292)$ \\
Sprachregion (Deutsch) & & & \\
Französisch & $0,657^{* * *}$ & $0,724 * * *$ & $0,589^{* * *}$ \\
& $(0,079)$ & $(0,105)$ & $(0,117)$ \\
Italienisch & $0,695^{* * *}$ & 0,230 & $1,20^{* * *}$ \\
& $(0,214)$ & $(0,268)$ & $(0,331)$ \\
Konstante & $5,21^{* * *}$ & $6,42^{* * *}$ & $4,25^{* * *}$ \\
$\mathrm{n}$ & 5.606 & 3.115 & 2.491 \\
Adj. ${ }^{2}$ (in Prozent): & 10,4 & 9,1 & 8,2 \\
\hline
\end{tabular}

Datenbasis: SHP 2008, gewichtet, eigene Berechnung (OLS Regression, unstandardisierte b-Koeffizienten, Referenzkategorie in Klammern, robuste Standardfehler in Klammern)

${ }^{a}$ Von den geschätzten schrittweisen (hierarchischen) Regressionsmodellen werden nur die Endmodelle präsentiert. Das Signifikanzniveau ist auf 5\% festgelegt. Auch unter Kontrolle der Variablen des Sozialkapitals, der kritischen Lebensereignisse und Sprachregion bleiben die Effekte der sozialstrukturellen Position (z.B. Geschlecht, Alter, Nationalität, Einkommen, Erwerbstätigkeit) stabil. Diese Robustheit bzgl. der ausgewiesenen sozialstrukturellen Effekte gilt ebenfalls für die geschätzten Teilmodelle der Frauen und Männer (Modelle nicht ausgewiesen).

Koeffizient sig. zum $* 5 \%, * * 1 \%, * * * 0,1 \%$-Niveau

Die Modelle zeigen keine negativen Alterseffekte wie sie z. B. Simon und Nath (2004) für die USA nachgewiesen haben. Hingegen verdeutlichen die Analysen, dass im Gesamtmodell die 40- bis 49-Jährigen und die 50- bis 59-Jährigen häufiger über Traurigkeit berichten (Referenz: 18- bis 29-Jährige). Dies entspricht Befunden, die ein stärkeres emotionales und psychisches Unwohlsein und Depressionen am Ende des mittleren Alters (ab ca. 50 Jahren) bzw. am Ende der reproduktiven Phase nachweisen (vgl. zusammenfassend Mirowsky und Ross 1989). Interessanterweise ist ein Vorzeichenwechsel feststellbar, denn über 70-Jährige geben - im Vergleich zur Referenzkategorie der 18- bis unter 30-Jährigen - weniger häufig an, Traurigkeit zu empfinden (Ausnahme Modell Frauen). Dieser Effekt zeigt sich unter Kontrolle von kritischen Lebensereignissen (z. B. Todesfälle, chronische Krankheiten), die im Alter wahrscheinlicher sind, und anderen relevanten Variablen der sozialstrukturellen Position sowie Sozialkapital. Dies entspricht bisherigen Studien, die über einen negativen Zusammenhang zwischen Alter und der Emotion Ärger berichten (vgl. Simon und Nath 2004; Schieman 2006). Interaktionseffekte zwischen dem Lebensalter und kritischen Lebensereignissen sind nicht signifikant und werden aus diesem Grund nicht ausgewiesen. Dennoch besteht an dieser Stelle weite- 
rer Forschungsbedarf, um zwischen möglichen Alters- oder Kohorteneffekten im Erleben und Ausdruck von Emotionen zu differenzieren.

Betrachtet man den Familienstand, so deuten die Modelle darauf hin, dass keine Unterschiede zwischen Verheirateten und Personen, die in einer Partnerschaft leben, bestehen. Bisherige Studien fokussieren primär auf den positiven Effekt des Heiratsstatus auf das emotionale Erleben (vgl. Mirowsky und Ross 1989; Simon und Nath 2004). Der Effekt der Heirat kann jedoch auf die vorhandene Partnerschaft als erklärenden Faktor zurückgeführt werden (vgl. auch Budowski et al. 2009). Singles geben im Vergleich zu Verheirateten häufiger an, traurig zu sein. Der Single-Effekt ist jedoch nicht für Frauen nachweisbar. In allen Modellen haben Kinder im Haushalt bzw. der Elternstatus keinen signifikanten Einfluss auf die Häufigkeit von Traurigkeit.

Bestehen Unterschiede zwischen der schweizerischen und ausländischen Bevölkerung im Erleben von Traurigkeit? Die Befunde bestätigen die These einer höheren emotionalen Belastung von Migrantinnen und Migranten in der Aufnahmegesellschaft. Zudem sind auch kulturelle Unterschiede im Erleben und Berichten von Emotionen denkbar. Personen, die neben der Schweizer Nationalität noch eine zweite Nationalität haben, sowie Nicht-Schweizerinnen und Schweizer (d.h. Personen mit ,,anderer Nationalität“) geben häufiger an, Traurigkeit zu empfinden. Dieser Befund erweist sich unter Kontrolle des sozioökonomischen Status und des Sozialkapitals als stabil. ${ }^{3}$

Romands besonders anfällig auf Depressionen lautet eine Schlagzeile des Schweizer Tagesanzeiger vom 9. November 2010 (vgl. Städler 2010). Dieser Zusammenhang wurde aufgrund der häufigeren Attestierung einer Depression vonseiten der Westschweizer Hausärztinnen und Hausärzte getroffen. Diese regionalen Effekte lassen sich nun auch für das Gefühl der Traurigkeit in der Romandie nachweisen, denn die Modelle zeigen signifikante Einflüsse der Sprachregion. Unter Kontrolle von relevanten sozialen Merkmalen wie sozioökonomischer Status, Sozialkapital oder kritischen Lebensereignissen berichten Bewohnerinnen und Bewohner der französisch- und italienischsprachigen Schweiz über ein häufigeres Traurigkeitsempfinden. An dieser Stelle können unterschiedliche Erklärungen angeführt werden, die sowohl auf den Minoritätenstatus als auch auf kulturelle Unterschiede rekurrieren. Zum einen ist ein schlechteres emotionales Wohlbefinden in der Romandie und im Tessin denkbar. Damit verbunden wären eine stärkere Anlehnung an und Zugehörigkeitsgefühle der französisch- und italienischsprachigen Schweizerinnen und Schweizer für ihre jeweiligen Nachbarländer Frankreich und Italien, was eine größere Distanz zur Deutschschweiz und Gefühle von Exklusion und Entfremdung im eigenen Land infolge dieser Minderheitenposition zur Folge haben könnte. Gestützt wird diese Vermutung durch kulturpsychologische Studien (vgl. Hofstede 2001; McCrae und Allik 2002). Ethnisch und historisch miteinander verbundene Länder weisen ähnliche Persönlichkeitsprofile auf. Sprachähnlichkeit und geografische Nähe sind Korrelate kultureller Ähnlichkeit (vgl. Roose 2010). Denkbar wäre zum anderen auch, dass es - verglichen mit der Deutschschweiz - einen anderen „Schwellenwert“ für das Empfinden von Traurigkeit gibt. Dieses Argument fokussiert auf kulturelle Interpretationsprozesse

3 Aufgrund der geringen Fallzahlen können die einzelnen Herkunftsländer nicht differenziert in die Analyse aufgenommen werden. 
und die Variabilität der Bedeutungen von Traurigkeit, tristesse und triztessa. ${ }^{4}$ Die Etymologie des deutschen Adjektivs „,traurig“ verweist dabei auf ein Gefühl „,von Trauer erfüllt, betrübt, betrüblich, schmerzlich, jämmerlich“ (vgl. Duden 2001). Eventuell unterscheiden sich die ,starke“ Definition von Traurigkeit und die jeweiligen Bedeutungen der französischen und italienischen Begriffe „tristesse“ und „triztessa“. Daneben können weitere Begründungen angeführt werden, die sich auf Hochschilds Expressionsnormen stützen, z. B. eine generell höhere emotionale Expressivität bzw. einen offeneren Umgang mit und Kommunikation von Emotionen. Eine niedrigere „Schwelle“ für das Empfinden und den Ausdruck von Traurigkeit, die individuelle Aufmerksamkeit ihr gegenüber sowie die Bereitschaft, über Emotionen Auskunft zu geben, könnten Erklärungen für die Befunde in der Romandie und im Tessin sein. Die Spezifizierung des Zusammenhangs zwischen Sprachregion und Emotionen erfordert jedoch noch weitere Forschungen und kann an dieser Stelle nicht abschließend geklärt werden.

In Erweiterung der bisherigen Studien wurden zusätzlich Einflüsse des Sozialkapitals sowie kritischer Lebensereignisse geprüft. Es lässt sich ein negativer Effekt der Netzwerkgröße feststellen (Ausnahme Modell Frauen). Je größer das persönliche Netzwerk und damit die Anzahl der engen Verwandten, Bekannten, Freundinnen und Freunde, desto weniger häufig wird über Traurigkeit berichtet. Damit bestätigen sich die Annahmen des Ressourcenkonzeptes der soziologischen Stresstheorie. Die externe Einbindung in Form einer Vereinsmitgliedschaft hat keinen Einfluss. In allen Modellen haben kritische Lebensereignisse als ,exogene Schocks“ (vgl. Ormel et al. 1999) signifikante Effekte auf die Häufigkeit der berichteten Traurigkeit. Das Konzept der „,normalen Traurigkeit“ (vgl. Horwitz und Wakefield 2007) ist definiert als emotionale Reaktion auf Veränderungen des sozialen und individuellen Kontextes, insbesondere Verlustereignisse (Tod Nahestehender, Ende einer engen Beziehung, chronische Krankheit). Dieses Konzept wird durch die vorliegende Studie empirisch bestätigt. Dennoch muss hervorgehoben werden, dass auch unter Kontrolle dieser kritischen Lebensereignisse Zusammenhänge zwischen der sozialstrukturellen Position und Traurigkeit stabil sind.

Abschließend sollen die Unterschiede zwischen dem Frauen- und Männermodell betrachtet werden, um mögliche Interaktionseffekte zu identifizieren. Es zeigen sich eindeutige Geschlechterdifferenzen in Bezug auf den Singlestatus und das Einkommen. Für Frauen lässt sich ein Zusammenhang zwischen der Höhe des Einkommens und Traurigkeit nachweisen, während der Einfluss des Singlestatus lediglich für Männer festzustellen ist. Frauen mit mittlerem Bildungsniveau berichten weniger häufig, traurig zu sein, als Frauen mit niedrigem Bildungsstatus. Ein weiterer Unterschied zeigt sich mit Blick auf die Sprachregionen. Sowohl in der italienisch- als auch in der französischsprachigen Schweiz geben Männer an, häufiger traurig zu sein (im Vergleich zur deutschsprachigen

4 Ein methodisches Artefakt aufgrund unterschiedlicher Übersetzungen und Frageformulierungen kann ausgeschlossen werden. Traurigkeit wurde in der französischen und italienischen Version des Fragebogens jeweils mit „tristesse“ und „triztessa“ übersetzt. Folgende Frageformulierungen wurden gewählt: 1) „A quelle fréquence ressentez-vous en général les émotions suivantes (tristesse), si 0 signifie ,jamais“ et 10 ,toujours“?" 2) „Con quale frequenza, di solito, Lei sperimenta le emozioni seguenti (triztessa), se 0 significa ,mai' et 10 ,sempre“?“" (vgl. http:// www.swisspanel.ch/spip.php?rubrique135\&lang=de. Zugegriffen: 22. Juni 2012). 
Schweiz), während dies nur auf Frauen aus der französischsprachigen Schweiz zutrifft. Dieser Befund könnte auf unterschiedliche kulturelle Gefühls- und Expressionsnormen für Männer in diesen Sprachregionen zurückgeführt werden, kann an dieser Stelle jedoch nicht vollständig geklärt werden. ${ }^{5}$

\section{Fazit}

Eine soziologische Studie über die gesellschaftliche Verteilung von Emotionen ermöglicht einen Perspektivenwechsel in der Betrachtung von Traurigkeit und hebt ihre soziale und kulturelle Variabilität hervor. Aus methodischer Sicht bleibt anzumerken, dass in dieser Untersuchung die emotionalen Konsequenzen objektiver Soziallagen, operationalisiert anhand soziodemografischer Variablen, analysiert wurden. Subjektive Einschätzungen und individuelle kognitive Bewertungen von Situationen sind aufgrund der Analyse von Survey-Daten nicht Gegenstand der Untersuchung. Diese Mechanismen können nur theoretisch vermutet werden. So werden Interpretationsleistungen von Individuen unterstellt, diese können jedoch nicht empirisch geprüft werden. Selbstauskünfte über die Häufigkeit des Traurigkeitsempfindens stellen zudem nur einen Indikator für emotionale Erfahrungen dar. Sie unterliegen Gefühls- und Expressionsnormen und damit der Gefahr, sozial wünschenswerte Antworttendenzen zu produzieren (Thoits 1989, S.339). In Anlehnung an von Scheve (2009, S.348) kann vermutet werden, dass ,diese Normen [...] weitgehend mit den unbewussten affektiven Reaktionen übereinstimmen, diese Reaktionen lassen sich [...] mit dem gängigen Instrumentarium jedoch kaum nachweisen. " Auch konnte zwischen dem tatsächlichen Erleben und dem verbalen Ausdruck von Emotionen in Bevölkerungsumfragen nicht explizit differenziert und keine Aussage über die Intensität der empfundenen Traurigkeit getroffen werden.

Trotz dieser Beschränkungen und datentechnischen Restriktionen leistet die vorliegende Studie einen Beitrag zur Analyse des Zusammenhangs zwischen Sozialstruktur und Emotionen, im Speziellen zu sozialen Korrelaten und zur gesellschaftlichen Verteilung von spezifischen Emotionen. Survey-Daten ermöglichen es, repräsentative Aussagen über Häufigkeit und Verteilung von Traurigkeit innerhalb der Gesellschaft zu treffen. Die Studie ist somit anschlussfähig an vertiefende Untersuchungen über Wahrnehmungen und Bewertungen von (Verlust-)Situationen, Gefühlsmanagement von Traurigkeit, Veränderungen von Traurigkeit im Lebenslauf sowie Intensität der erlebten Traurigkeit, die jedoch eine andere Methodik (z. B. qualitative Verfahren) erfordern.

Die Befunde heben die Bedeutung von Emotionen als wichtigem Forschungsgegenstand in der deutschsprachigen Soziologie hervor. Traurigkeit ist nicht zufällig in der Schweizerischen Bevölkerung verteilt, sondern weist soziale Regelmäßigkeiten und Ungleichheiten auf. Die Studie stellt damit einen wichtigen Beitrag zur Epidemiologie von Emotionen (vgl. Thoits 1989) dar. Die Ergebnisse dieser Studie geben Hinweise auf eine emotionale Ungleichheit innerhalb der Gesellschaft. Es lassen sich Tendenzen einer ungleichen Verteilung der Emotion Traurigkeit erkennen, die jedoch durch weitere Ana-

5 Die Befunde für Befragte der italienischsprachigen Schweiz sind aufgrund geringer Fallzahlen eher vorsichtig zu interpretieren und erfordern weitere vertiefende Analysen. 
lysen abgesichert werden müssen. Interpretiert man Traurigkeit als einen Indikator für Lebenszufriedenheit, so lassen sich Benachteiligungen für spezifische soziale Gruppen identifizieren. Der Beitrag bestätigt Variationen des Traurigkeitsempfindens entlang sozialstruktureller Kategorien wie Geschlecht, sozioökonomischer Status, Alter oder Nationalität. Ökonomische Restriktionen (Einkommen) als Korrelate von negativen Emotionen können ebenfalls für die Schweiz bestätigt werden. Die Befunde zu den geschlechtsund kulturell bedingten Variationen von Traurigkeit verweisen auf andere theoretische Erklärungen. Ursachen für diese Zusammenhänge werden vielmehr in einer unterschiedlichen Gefühlssozialisation, kulturellen Gefühls- und Expressionsnormen (Hochschild) und Traurigkeitsdefinitionen vermutet. Gleichzeitig können aber auch das Geschlecht und die ethnische Herkunft als Statusmerkmale im Kemper'schen Sinne interpretiert werden. Signifikante Unterschiede in der Häufigkeit des Traurigkeitsempfindens zeigen sich ebenfalls zwischen den Schweizer Sprachregionen. Bewohnerinnen und Bewohner der Romandie und des Tessins geben an, häufiger Traurigkeit zu empfinden. Allerdings besteht an dieser Stelle noch weiterer Forschungsbedarf. Darüber hinaus wurden kritische Lebensereignisse und Sozialkapital als bisher vernachlässigte Determinanten in der soziologischen Emotionsforschung geprüft. Unter Kontrolle dieser externen Bedingungen sind die zuvor beschriebenen Zusammenhänge zwischen dem sozialen Status, Alter, Geschlecht oder Sprachregion und Traurigkeit stabil. Gleichzeitig kann die vorliegende Studie die Bedeutung von sozialen Netzwerken als soziale Ressourcen für das emotionale Wohlbefinden bestätigen. In Übereinstimmung mit der soziologischen Stresstheorie kann ebenfalls der Einfluss von kritischen Lebensereignissen auf die Häufigkeit von Traurigkeit nachgewiesen werden. Die Studie sensibilisiert somit für die sozial ungleiche Verteilung von Emotionen und bestätigt den Zusammenhang zwischen der sozialstrukturellen Position, zur Verfügung stehenden Ressourcen, externen Ereignissen und dem Gefühl der Traurigkeit innerhalb einer Gesellschaft.

Danksagung: Ich danke den beiden anonymen GutachterInnen für hilfreiche Anregungen.

\section{Literatur}

Aneshensel, Carol S., Carolyn M. Rutter, und Peter A. Lachenbruch. 1991. Social structure, stress, and mental health: competing conceptual and analytic models. American Sociological Review 56:166-178.

Badura, Bernhard, und Holger Pfaff. 1989. Stress, ein Modernisierungsrisiko? Mikro- und Makroaspekte soziologischer Belastungsforschung im Übergang zur postindustriellen Zivilisation. Kölner Zeitschrift für Soziologie und Sozialpsychologie 41:644-668.

Bonanno, George A., Laura Goorin, und Karin G. Coifman. 2010. Sadness and grief. In: Handbook of emotions, Hrsg. Michael Lewis, Jeannette M. Haviland-Jones, und Lisa Feldman Barrett, 797-810. New York: The Guilford Press.

Bowlby, John. 1980. Attachment and loss. Vol. III. Loss. Sadness and depression. New York: Basis Books.

Brody, Leslie R. 1997. Gender and emotion. Beyond stereotypes. Journal of Social Issues 53:369-394. 
Budowski, Monica, Maurizia Masia, und Robin Tillmann. 2009. Psychological health: An analysis of the intersection of cumulative disadvantage and partnership events. Swiss Journal of Sociology 35:357-376.

Carstensen, Laura L. 1991. Selectivity theory: Social activity in life-span context. In: Annual Review of Geriatrics and Gerontology, Hrsg. Warner K. Schaie, 195-217. New York: Springer.

Carstensen, Laura L. 1992. Social and emotional patterns in adulthood: Support for the socioemotional selectivity theory. Psychology and Ageing 7:331-338.

Duden. 2001. Das Herkunftswörterbuch. Etymologie der deutschen Sprache. Band 7. Mannheim: Bibliographisches Institut \& F. A. Brockhaus Setzerei GmbH.

Filipp, Sigrun-Heide. 1981. Kritische Lebensereignisse. München: Urban und Schwarzenberg.

Flam, Helena. 2002. Soziologie der Emotionen. Stuttgart: UTB.

Freitag, Markus. 2004. Schweizer Welten des Sozialkapitals. Empirische Untersuchungen zum sozialen Lernen in Regionen und Kantonen. Swiss Political Science Review 10:87-118.

Hochschild, Arlie R. 1979. Emotion work, feeling rules and social structure. American Journal of Sociology 85:551-575.

Hochschild, Arlie R. 2006. Das gekaufte Herz. Zur Kommerzialisierung der Gefühle. Frankfurt am Main: Campus.

Hofstede, Geert H. 2001. Culture's consequences: Comparing values, behaviors, institutions, and organizations across nations. Thousand Oaks: Sage.

Hopkins, Deborah, Jochen Kleres, Helena Flam, und Helmut Kuzmics. 2009. Theorizing Emotions: Sociological Explorations and Applications. New York: Campus.

Horwitz, Allan V., und Jerome C. Wakefield. 2007. The loss of sadness. How psychiatry transformed normal sorrow into depressive disorder. Oxford: Oxford University Press.

Hurrelmann, Klaus. 2000. Gesundheitssoziologie. Eine Einführung in sozialwissenschaftliche Theorien von Krankheitsprävention und Gesundheitsförderung. 4. Aufl. Weinheim: Juventa Verlag.

Kemper, Theodore D. 1981a. Auf dem Wege zu einer Theorie der Emotionen: Einige Probleme und Lösungsmöglichkeiten. In: Logik des Herzens. Die soziale Dimension der Gefühle, Hrsg. Gerd Kahle, 134-154. Frankfurt am Main: Suhrkamp.

Kemper, Theodore D. 1981b. Social constructionist and positivist approaches to the sociology of emotions. American Journal of Sociology 87:336-362.

Kemper, Theodore D. 1991. Predicting emotions from social relations. Social Psychology Quarterly 54:330-342.

Lennon, Mary C. 1989. The structural contexts of stress. an invited response to pearlin. Journal of Health and Social Behaviour 30:261-268.

Leventhal, Allan, und Christophe Martell. 2006. The Myth of Depression as Disease: Limitations and Alternatives to Drug Treatment. Westport, Conn.: Praeger Publishers.

McCrae, Robert R., und Juri Allik. 2002. The Five-Factor Model of personality across cultures. New York: Kluwer Academic.

McLanahan, Sara, und Julia Adams. 1987. Parenthood and psychological well-being. Annual Review of Sociology 13:237-257.

McLanahan, Sara, und Julia Adams. 1989. The effects of children on adults' psychological wellbeing: 1957-1976. Social Forces 68:124-146.

Mirowsky, John, und Catherine E. Ross. 1986. Social patterns of distress. Annual Review of Socio$\log y$ 12:23-45.

Mirowsky, John, und Catherine E. Ross. 1989. Social Causes of Psychological distress. New York: Aldine de Gruyter.

Mirowsky, John, und Catherine E. Ross. 1995. Sex differences in distress: real or artifact? American Sociological Review 60:449-468.

Neckel, Sighard. 1999. Blanker Neid, blinde Wut? Sozialstruktur und kollektive Gefühle. Leviathan 27:145-165.

Ormel, Johan, Siegwart Lindenberg, Nardi Steverink, und Lois M. Verbrugge. 1999. Subjective well-being and social production functions. Social Indicators Research 46:61-90. 
Pearlin, Leonard I. 1989. The sociological study of stress. Journal of Health and Social Behaviour 30:241-256.

Riches, Gordon, und Pam Dawson. 2000. An intimate loneliness: Supporting bereaved parents and siblings. Buckingham: Open University Press.

Roose, Jochen. 2010. Der Index kultureller Ähnlichkeit. Konstruktion und Diskussion. Berliner Studien zur Soziologie Europas. BSSE-Arbeitspapier Nr. 21. Berlin: Freie Universität Berlin.

Rüesch, Peter, und Patrik Manzoni. 2003. Psychische Gesundheit in der Schweiz. Monitoring. Neuchâtel: Schweizerisches Gesundheitsobservatorium.

Schieman, Scott. 2006. Anger. In: Handbook of the sociology of emotions, Hrsg. Jan E. Stets und Jonathan H. Turner, 493-515. New York: Springer.

Simon, Robin W. 2002. Revisiting the relationships among gender, marital status, and mental health. American Journal of Sociology 107:1065-1096.

Simon, Robin W., und Kathryn Lively. 2010. Sex, anger and depression. Social Forces 88:1543-1568.

Simon, Robin W., und Leda E. Nath. 2004. Gender and emotion in the United States: do men and women differ in self-reports of feelings and expressive behavior? American Journal of Socio$\log y$ 109:1137-1176.

Städler, Iwan. 2010. Romands besonders anfällig auf Depressionen. Tagesanzeiger, http:// www.tagesanzeiger.ch/schweiz/standard/Romands-besonders-anfaellig-auf-Depressionen/ story/27183549, 05.05.2011. Zugegriffen: 9. Nov. 2010.

Stevenson, Betsey, und Wolfers Justin. 2009. The paradox of declining female happiness. American Economic Journal: Economic Policy 1:190-225.

Stroebe, Wolfgang, und Margaret S. Stroebe. 1987. Bereavement and health: the psychological and physical consequences of partner loss. Cambridge: Cambridge University Press.

Stylianos, Stanley K., und Mary L.S. Vachon. 2006. The role of social support in bereavement. In Handbook of Bereavement. Theory, research, and intervention, Hrsg. Margaret S. Stroebe, Wolfgang Stroebe und Robert O. Hansson, 397-410. Cambridge u. a.: Cambridge University Press.

Thoits, Peggy A. 1989. The sociology of emotions. Annual Review of Sociology 15:317-342.

Turner, Jonathan H. 2007. Human emotions. A sociological theory. London: Routledge.

Turner, Jonathan H., und Jan E. Stets. 2005. The Sociology of emotions. Cambridge: Cambridge University Press.

Vester, Heinz-Günter. 1991. Emotion, Gesellschaft und Kultur. Opladen: Westdeutscher Verlag.

von Scheve, Christian. 2009. Emotionen und soziale Strukturen. Die affektiven Grundlagen sozialer Ordnung. Frankfurt am Main: Campus.

von Scheve, Christian. 2012. Die sozialen Grundlagen der Emotionsentstehung: Kognitive Strukturen und Prozesse. In Emotionen, Sozialstruktur und Moderne, Hrsg. Annette Schnabel und Rainer Schützeichel, 115-137. Wiesbaden: Springer VS.

Winkel, Heidemarie. 2004. „Reden ist aber gerade das Entscheidende ...“ - Trauerberatung und die Ausdifferenzierung semantischer Strukturen der Problematisierung individuellen Leids. In Die beratende Gesellschaft, Hrsg. Rainer Schützeichel und Thomas Brüsemeister, 181-203. Wiesbaden: VS Verlag für Sozialwissenschaften.

Wolf, Christof. 2004. Egozentrierte Netzwerke. Erhebungsverfahren und Datenqualität. In Methoden der Sozialforschung. Hrsg. Andreas Diekmann. Sonderheft der Kölner Zeitschrift für Soziologie und Sozialpsychologie 44:244-273.

Yu, Yan, und David R. Williams. 2006. Socioeconomic Status and Mental Health. In Handbook of the Sociology of Mental Health, Hrsg. Carol S. Aneshensel und Jo C. Phelan, 151-166. New York: Springer.

Nina R. Jakoby, Dr. phil., 1996-2001 Studium der Diplom-Soziologie an der Universität Trier, 2007 Promotion an der RWTH Aachen University, seit 2008 Oberassistentin und Lehrbeauftragte am Soziologischen Institut der Universität Zürich. 